\title{
Model of dynamic tariffing Microgrid's electricity consumption in local energy markets
}

\author{
V. Y. Zhuikov, I. Y. Boiko, S. P. Denysiuk \\ National Technical University of Ukraine \\ "Igor Sikorsky Kyiv Polytechnic Institute", Kyiv, Ukraine \\ Corresponding author E - mail: catbug1488@gmail.com
}

Paper received 04.02.21; Accepted for publication 16.02.21.

\section{https://doi.org/10.31174/SEND-NT2021-250IX31-11}

Abstract. In order to calculate the cost of electricity, the urgent task is to create a dynamic model that will calculate the change in the cost of electricity with a dynamic change in its level. The article considers an example of a local system based on an autonomous diesel generator. The resulting model allows us to study the change in cost indicators of the economic system when the level of electricity generated by the generator is changed. The obtained equation allows to calculate the price of the base part of energy depending on the studied level of energy produced by the generator and its value at a certain point of rest.

Keywords: Microgrid; local generating system; dynamic model; Fisher equation.

Introduction. In December 2019, the European Commission presented The Green European Deal, an ambitious program that describes the transition to a climate-neutral, circular economy by 2050 . This program symbolizes significant changes in EU policy.

The new political and energy doctrine of the European Union, adopted in December 2020, describes the rules of reforming the European Union in the energy sector and the creation of a trans-European energy network - TEN-E. The EU's priorities are shifting from fossil fuels to clean generation to ensure a fully decarbonised economy by 2050. A Smart Grid is defined in the TEN-E Regulation (European Commission) as an electricity network that can integrate in a cost-efficient manner the behavior and actions of all users connected to it, including generators, consumers and those that both generate and consume, in order to ensure an economically efficient and sustainable power system with low losses and high levels of quality, security of supply and safety. The TEN-E Regulation has identified smart grid deployment as one of 12 trans-European energy infrastructure priority corridors and areas.

Modern European policy Smart Grid provides the following priority areas: the introduction of renewable energy sources (RES); construction of local systems (Microgrid) for various purposes; new business processes in liberalized local markets with the participation of electricity aggregators. The problem of system flexibility needs to be solved, the role of aggregators at all levels in the formation of energy as service policy is growing: national, regional, local (combination of several energy supply systems) and individual (specific system containing active prosumer or virtual power plant, (VPP)). In addition to new technical and technological solutions, there is a need to improve the mechanisms of Microgrid in local markets. This encourages the consideration of the operation of Microgrid facilities in liberalized local markets, the implementation of flexible tariff policies, in particular, dynamic tariffing procedures. If at the national level the price correction (change of tariffs) is carried out at intervals of 15 minutes (Germany) and 5 minutes (USA), then at the local and individual levels, changes in the price of generation and consumption in Microgrid should be considered at a much shorter interval.

Using a shorter billing interval $\Delta t$, even to fractions of minutes allow: more accurately optimize energy processes in the system; to form adequate price signals that will allow to make more accurate settlements, to conduct technical and commercial accounting with greater accuracy; the ability to refine the signals of the control system by introducing into the control scheme of the economic component for the use of primary fuel, production and consumption of electricity. At the same time, a more accurate summing up of the balance of energy components in the modes of generation, accumulation and consumption is carried out, when it is possible to distinguish energetically constant states of the system operation and take into account the bidirectionality of electricity transmission. At the same time, there is a second level of cash flow balancing associated with the first, energy.

A brief overview of publications on the topic. A significant amount of scientific research and publications is devoted to the problem of Microgrid's functioning in local energy markets. Thus, as a result of research $[1,2]$, have been developed integrated models of consumer response and the theory of tariff price forecasting in dynamic conditions, which are created through dynamic tariffing of electricity. In [3] is presented a new algorithm for planning restrictions imposed for network security reasons on one basic normally functioning system and the formation of a list of possible unforeseen circumstances (linear excess, block loss, load increase) of the system. A mathematical model of distribution system planning was also created, which includes three variants of system expansion planning [4]. The real-time tariffing scheme of the power generator presented in [5] is based on the current states of the system, which are forecast in hourly scenarios, and the calculated tariff prices for these states. The disadvantage of this model is its linearity. One of the approaches to creating dynamic tariffing is also the regulation of tariffing mechanisms based on optimal management [6]. In the model [7] the functions are linear and do not take into account the dynamic change of the generated power.

The disadvantages of the considered models [1, 2, 5, 7] are the use of linearized characteristics and a significant sampling interval over time.

The purpose of the work is to build a model of dynamic tariffing and an algorithm for implementing this model for Microgrid systems, which should ensure the optimal functioning of such systems in today's local markets.

Materials and methods. Consider the construction of a mathematical model for determining the price of the base part of electricity, which can be used for tariff pricing for a 
fairly short time interval for a single generator, such as a diesel generator, and the consumer. The system under consideration is essentially nonlinear, but to build a mathematical model of process analysis in "small", we use the description of processes in this system by linear algebraic and differential equations [8].

Determining the cost of electricity for dynamic tariffing depends on two components: economic and technical. We analyze the economic component on the basis of Fisher equation, which describes the balance of the economic system [9]:

$$
M \cdot V=C_{Q} \cdot Q \text {, }
$$

where $M-$ money supply, which makes one revolution for some time $T_{V}, \quad V$ - the number of revolutions of the money supply for the studied period of time; $C_{Q}$ - product price; $Q$ - number of products.

To calculate the cost of energy generated, the right side of expression (1) should include all costs associated with energy generation, such as primary fuel costs, for materials, depreciation, wages, and others determined by the operating characteristics of the generating equipment. For simplicity and clarity of presentation of material we will lower all expenses, except for expenses for fuel which is necessary for production of the electric power by the certain generator (in our case the diesel generator).

Taking as a base value the part of energy expressed in $(W \cdot s e c)$ we rewrite the Fisher equation in the form:

$$
M \cdot V=C_{B} \cdot B+C \cdot W
$$

where $C, C_{B}$ - the cost of the base part of energy and unit of fuel; $W, B$ - volumes of consumed electricity and primary fuel.

For further operation with the cost of electricity, we introduce the coefficient $\sigma=C_{B} / C$, equation (2) writes with increments $\Delta$ relative to variabled values $M, C, B, W$. If we neglect the members of the higher (second) order of minority, it is obtained:

$$
\begin{gathered}
(M+\Delta M) \cdot V=C \cdot \sigma \cdot B+C \cdot \sigma \cdot \Delta B+\Delta C \cdot \sigma \cdot B+ \\
C \cdot W+C \cdot \Delta W+\Delta C \cdot W .
\end{gathered}
$$

Subtracting from equation (3) equation (2) is obtained: $\Delta C \cdot(W+\sigma \cdot B)=V \cdot \Delta M-C \cdot \sigma \cdot \Delta B-C \cdot \Delta W$.

Going from the operator $\Delta$ to $\mathrm{d} / \mathrm{dt}$ for increments in small functions $C(t)$ and $W(t)$ and bringing the left and right parts of the equation to the same dimension given the time of change of increments, obtained:

$$
\begin{aligned}
& \quad \frac{d C(t)}{d t}=\frac{V \cdot \Delta M}{(W(t)+\sigma \cdot B(t)) \cdot \tau}-\frac{C_{B} \cdot \Delta B}{(W(t)+\sigma \cdot B(t)) \cdot \tau}-\frac{C(t)}{W(t)+\sigma \cdot B(t)} . \\
& \frac{d W(t)}{d t}
\end{aligned}
$$

where $\tau$ - the time of deviation of the amount of electricity and its cost relative to the selected values $W_{0}$ and $C_{0}$ at point of stability.

If considering the operation of the generating system for some time interval $[0, T]$, when increments $\frac{d C(t)}{d t}, \frac{d W(t)}{d t} \neq$ 0 , then expression (5) can be converted to the form: $\frac{d C(t)}{d t}=\frac{V \cdot \Delta M-C_{B} \cdot \Delta B}{(W(t)+\sigma \cdot B(t)) \cdot \tau}-\frac{C(t)}{W(t)+\sigma \cdot B(t)} \cdot \frac{d W(t)}{d t}$.

After performing algebraic transformations, relation (5) can be represented in the form:

$$
\begin{aligned}
& \frac{1}{C(\mathrm{t})} \cdot d C(t)=\frac{V \cdot \Delta M-C_{B} \cdot \Delta B}{(W(t)+\sigma \cdot B(t)) \cdot \tau \cdot C(t)} \cdot d t-\frac{1}{W(t)+\sigma \cdot B(t)} . \\
& d W(t) .
\end{aligned}
$$

where $W(t)$ i $C(t)$ - the value of electricity produced and the cost of its base part at the investigated time moment;
$W_{0}$ - the value of energy generated by the generator at point of stability and $C_{0}-$ its cost; $T$ - the duration of the studied time interval.

To solve equation (7), the integration of the left part is performed within $C(t)$ to $C_{0}$. The first term of the right part from 0 to $T$, the second part from $W(t)$ to $W_{0}$. As a result of integration, it is received:

$$
\begin{aligned}
& \int_{C(t)}^{C_{0}} \frac{1}{C(\mathrm{t})} \cdot d C(t)=\int_{0}^{T} \frac{V \cdot \Delta M-C_{B} \cdot \Delta B}{(W(t)+\sigma \cdot B(t)) \cdot \tau \cdot C(t)} \cdot d t- \\
& \int_{W(t)}^{W_{0}} \frac{1}{W(t)+\sigma \cdot B(t)} \cdot d W(t) .
\end{aligned}
$$

The following replacements have been introduced:

$$
\left(V \cdot \Delta M-C_{B} \cdot \Delta B\right)=A . \quad((W(t)+\sigma \cdot B(t)) \cdot
$$

$C(t))=B$, if the value of the functions $W(t), B(t), C(t)$ taken averaged over the interval $[0 ; T]$.

$$
\int_{0}^{T} \frac{V \cdot \Delta M-C_{B} \cdot \Delta B}{(W(t)+\sigma \cdot B(t)) \cdot \tau \cdot C(t)} \cdot d t=\int_{0}^{T} \frac{A}{B \cdot \tau} \cdot d t .
$$

On condition that $T=\tau$, the definite integral is equal to: $\frac{A \cdot T}{B \cdot T}-\frac{A \cdot 0}{B \cdot T}=\frac{A}{B}$. Note that according to equation (8), the duration of the interval $[0, T]$ must be equal $\tau$, because during this time there are deviations of energy values and the value of its base unit from the values at the point of of stability.

Replacement performed: $\frac{A}{B}=D$. Then:

$$
\int_{C(t)}^{C_{0}} \frac{1}{C(\mathrm{t})} \cdot d C(t)=D-\int_{W(t)}^{W_{0}} \frac{1}{W(t)+\sigma \cdot B(t)} \cdot d W(t), \quad \text { and }
$$

accordingly:

$$
\begin{aligned}
& \ln \left(C_{0}\right)-\ln (C(t))=-\left(\ln \left(W_{0}+\sigma \cdot B(t)\right)-\right. \\
& \ln (W(t)+\sigma \cdot B(t)))+D \\
& \ln \left(\frac{C(t)}{C_{0}}\right)=\ln \left(\frac{W_{0}+\sigma \cdot B(t)}{(W(t)+\sigma \cdot B(t)) \cdot e^{D}}\right) .
\end{aligned}
$$

Performing the potentiation of both parts of equation (10) obtained expression (11), which determines the dynamic change in the cost of the base part of the energy when changing the volume of its production:

$$
C(t)=\frac{W_{0}+\sigma \cdot B_{0}}{(W(t)+\sigma \cdot B(t)) \cdot e^{D \cdot T}} \cdot C_{0} .
$$

Taking into account that $T=\tau$ :

$$
C(t)=\frac{W_{0}+\sigma \cdot B_{0}}{(W(t)+\sigma \cdot B(t)) \cdot e^{\frac{V \cdot(W(t)+\sigma \cdot B(t)) \cdot C(t)}{(W B}}} \cdot C_{0} .
$$

Here and after, taken into account that the values of the functions $W(t), B(t), C(t)$ taken averaged over the studied interval, function (12) will look like:

$$
C(t)=\text { const } \cdot \frac{1}{e^{x}}
$$

where const $=\frac{\left(W_{0}+\sigma \cdot B_{0}\right) \cdot C_{0}}{(W(t)+\sigma \cdot B(t))}, \quad x=\frac{V \cdot \Delta M-C_{B} \cdot \Delta B}{(W(t)+\sigma \cdot B(t)) \cdot C(t)}$ are constants. Taking into account that $\sigma=\frac{C_{B}}{C}$, in the point of of stability $C=C_{0}$ and in study time $C=C(t)$ received:

$$
\text { const }=\frac{W_{0} \cdot C_{0}+C_{B} \cdot B_{0}}{W(t)+\sigma \cdot B(t)}, x=\frac{V \cdot \Delta M-C_{B} \cdot \Delta B}{W(t) \cdot C(t)+C_{B} \cdot B(t)} .
$$

Using the substitutions made, an expression is obtained to determine the value $C(t)$ :

$C(t)=\frac{W_{0} \cdot C_{0}+C_{B} \cdot B_{0}}{W(t)+\sigma \cdot B(t)} \cdot e^{-\frac{V \cdot \Delta M-C_{B} \cdot \Delta B}{W(t) \cdot C(t)+C_{B} \cdot B(t)}}$

Relation (13) for different cases of system operation can be simplified when in the equation of exchange a certain number of increments of values is equal to zero. Thus we will receive a necessity of performance of a condition $V$. $\Delta M=C_{B} \cdot \Delta B$, which allows to convert equation (7) to the form $\frac{d C(t)}{d t}=-\frac{C(t)}{W(t)+\sigma \cdot B(t)} \cdot \frac{d W(t)}{d t}$, from which it is obtained $\frac{d C(t)}{C(t)}=-\frac{d W(t)}{W(t)+\sigma \cdot B(t)}$. Thus it is checked that the gains $\frac{d C(t)}{d t}$, $\frac{d W(t)}{d t}$ must be equal to zero to perform equation (7) under 
the new conditions, and calculation $C(t)$ should be performed at the point of stability. For a state of stability can be noted that when $\frac{d C(t)}{d t}=0$ and $\frac{d W(t)}{d t}=0, V \cdot \Delta M=C_{B} \cdot \Delta B$ , which corresponds to the condition of economic balance, which is described by Fisher equation.

Within from $C(t), W(t)$ to $C_{0}, W_{0}$, where $W(t)$ i $C(t)-$ the value of produced electricity and the cost of its base part at the studied time, $W_{0}-$ the value of the the energy produced by the generator at point of stability, and $C_{0}-$ its cost, integrated both parts of the simplified equation (7), ie: $\int_{C(t)}^{C_{0}} \frac{1}{C(t)} \cdot d C(t)=-\int_{\mathrm{W}(\mathrm{t})}^{W_{0}} \frac{1}{W(t)+\sigma \cdot B(t)} \cdot d W(t)$, and accordingly:

$$
\begin{gathered}
\ln \left(C_{0}\right)-\ln (C(t))=-\left(\ln \left(W_{0}+\sigma \cdot B(t)\right)-\ln (W(t)+\right. \\
\sigma \cdot B(t))) .
\end{gathered}
$$

Performing the potentiation of both parts of equation (15), obtained expression (16), which determines the dynamic change in the cost of the base part of the energy when the volume of its production changes:

$C(t)=\frac{W_{0}+\sigma \cdot B_{0}}{W(t)+\sigma \cdot B(t)} \cdot C_{0}$.

Due to the fact that with small deviations $B(t)$ from the level $B_{0}$ in equation (16) it is sufficient to take into account only the value $W(t)$ in the denominator, which deviates slightly from the state of stability:

$C(t)=\left.\frac{W_{0 i}+\sigma \cdot B_{0 i}}{W(t)+\sigma \cdot B_{0 i}} \cdot C_{0 i}\right|_{W_{0 i} \epsilon \Delta W_{i}}$.

Therefore, the calculated expression (17) is obtained for the dynamic calculation of the cost of the base part of the electricity at small deviations from the point of stability. The using of expression (17) involves a preliminary division of the range of changes in levels of electricity consumption into zones, within which there is a current control of consumption of primary fuel and electricity generation at constant tariffs (relative to the point $W_{0}$ with $\operatorname{cost} C_{0}$ ). When leaving this zone, it is necessary to adjust tariffs for the cost of electricity.

The figure shows the $i-$ th interval of change in the generated energy.

For the above presentation of interval $W_{i} \equiv W_{0}, C_{i} \equiv C_{0}$.

The determination of the cost can be demonstrated by the example of its calculation for small deviations from some static level of energy generated at the output of an autonomous diesel generator type Teksan TJ138PE5C [15], namely, with deviations of $\pm 5 \%$ from the generation mode of $50 \%$ of the rated power. The diesel generator is designed for a power of $100 \mathrm{~kW}$. When generating $50 \%$ of the rated power, fuel consumption is $15.52 \mathrm{l} / \mathrm{h}$, that taking into account only the market value of diesel fuel costs $22.34 \cdot 15.52$ $=347 \mathrm{uah} / \mathrm{h}$, and received power level is $50 \mathrm{~kW} \cdot \mathrm{h}$. Then $C_{0 i}=\frac{347}{50}=6,9 \frac{u a h}{k W \cdot h}$. For example it was calculated $C(t)$ by expression (17), ie not taking into account the deviation of fuel consumption $B(t)$ from the fuel level $B_{0 i}$, and considering, when $B(t) \neq B_{0 i}$ that the expression (16). It is accepted that expressions $\sigma \cdot B_{0 i}$ and $\sigma \cdot B(t)$ are equal to the energy at the input of the generator for the selected operating mode.

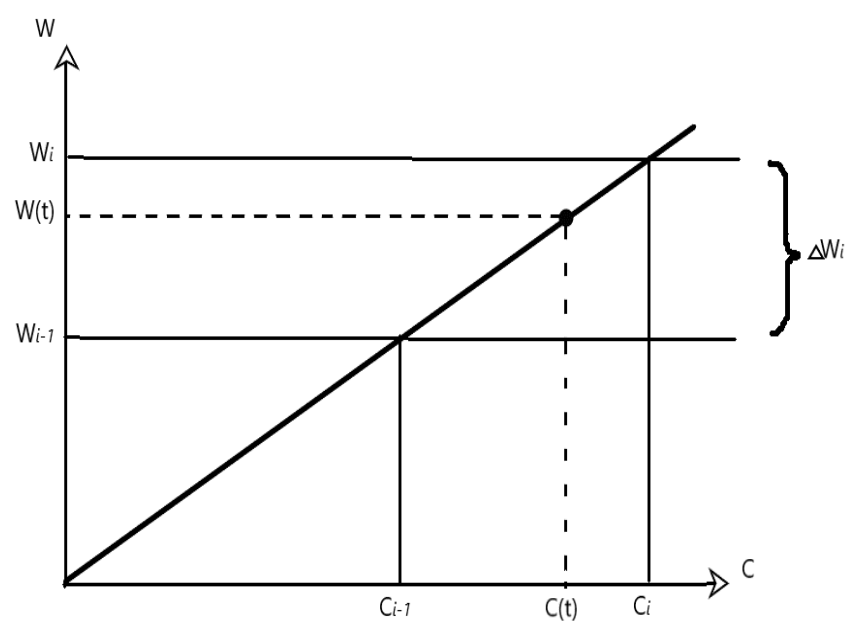

Figure - The studied interval

The values of fuel consumption and energy at the input of the generator will have the values given in table. Also in table. 1 shows the results of calculations of the cost of the base part of the energy in the nominal generation mode of $50 \mathrm{kWh}$ and with a deviation of $5 \%$ from the generation mode. In rates of fuel consumption liters must be converted to grams, using the conversion factor of fuel, and hours to seconds, since when creating per-second tariffing, rate of fuel consumption must be taken into account and paid every second. On the basis of the calculations was showed that the deviation from some small value set for the selected mode of generating energy tariff value will change accordingly: will increase with a decrease in the value of energy, and will fall with increasing in the value of energy.

\begin{tabular}{|c|c|c|c|c|c|c|c|c|}
\hline \multicolumn{4}{|c|}{ Calculation by expression (17) } & \multicolumn{4}{|c|}{ Calculation by expression (16) } & \multirow[b]{2}{*}{$\Delta(\%)$} \\
\hline Fuel consumption $\left(\frac{g}{s}\right)$ & $\begin{array}{c}\text { Input energy } \\
(k W \cdot h)\end{array}$ & $\begin{array}{c}\text { Output energy } \\
(k W \cdot h)\end{array}$ & $C(t)\left(\frac{\mathrm{uah}}{\mathrm{kW} \cdot \mathrm{h}}\right)$ & $\begin{array}{l}\text { Fuel consumption } \\
\qquad\left(\frac{g}{c}\right)\end{array}$ & $\begin{array}{c}\text { Input energy } \\
(k W \cdot h)\end{array}$ & $\begin{array}{l}\text { Output energy } \\
(k W \cdot h)\end{array}$ & $\left.C(t)\left(\frac{\mathrm{uah}}{\mathrm{kW} \cdot \mathrm{h}}\right)\right)$ & \\
\hline 3,62 & 154,2 & 47,5 & 6,98 & 3,44 & 146,5 & 47,5 & 7,29 & 4,25 \\
\hline 3,62 & 154,2 & 50 & 6,9 & 3,62 & 154,2 & 50 & 6,9 & 0 \\
\hline 3,62 & 154,2 & 52,5 & 6,81 & 3,8 & 161,9 & 52,5 & 6,57 & 3,52 \\
\hline
\end{tabular}

Table. 1. Calculation data

Given the fact that the output time of the diesel generator to the operating mode is greater than the interval of 1s. and the error $(\Delta(\%))$ between the calculations of the dynamic change $C(t)$ by two methods is about $4 \%$, in the future it is advisable to perform the calculation only by expression (17).

Also, for the given model was developed the algorithm of dynamic tariffing for Microgrid which works in an isolated mode. Let the load be connected to a meter that registers the change in the amount of energy received in dynamic mode. If there are several loads and each is connected to its own generator, which is equipped with a microcontroller $M C_{g i}$, and each load has its own built-in meter $M_{i}$. Also, each load has a microcontroller $M C_{l i}$.

Using of dynamic tariffing when changing the modes of operation of the generator should provide an adequate price for this system for consumers and producers of electricity.

The steps of the developed algorithm are given below:

1. At each time interval, data on the total amount of electricity produced by all generators is collected $W_{\Sigma}=$ $\sum_{i=1}^{n} W_{i}$. The time interval is selected from the technically 
acceptable conditions of dynamic change in the amount of electricity produced.

2. In asynchronous mode, each load microcontroller receives information about the amount of electricity consumed $W_{i}$ and calculates the weight coefficient $W_{w i}=\frac{W_{i}}{W_{\Sigma}}$, the cost of the base part of electricity $C_{i}$ according to the equation (17).

3. Microcontroller $M C_{g i}$ calculates the weight coefficient of the cost of the base part of electricity $C_{w i}=C_{0 i} \cdot W_{w i}$ for

4. Based on the calculated weight coefficients and obtained values of cost of base part of electricity $C_{i}$ the general microcontroller $M C$ determines the total cost of the base part of the electricity:

$$
C_{\Sigma}=\sum_{i=1}^{n} C_{\text {ваг } i} \cdot C_{i}
$$

5. The microcontroller of each load forms a word for the exchange protocol via the ethernet network with the server. The word indicates the weight coefficient of each generator. The network number $N_{i}$ is also written in each word, as well as the operating time of the corresponding generator $t_{i}$, the amount of generated energy $W_{i}$ and the calculated cost of the base part of the energy $C_{i}$.

6 . Based on the obtained data on the weight of this generator, the share of its use and the corresponding cost of the base part of the electricity are determined.

7. The common microcontroller $M C$ connected to the server generates a response word and sends it to the microcontroller $M C_{g i}$ of this particular generator.

8. Word containing the command to increase or decrease the amount of electricity generated based on $W_{i}$, as well as the new calculated cost of the base part of the electricity $C_{n e w i}=C_{i} \cdot C_{w i}$ based on data of the deviation of the level of generated energy and the weight coefficient of the cost of the base part of the energy $C_{w i}$ this generator.

Features of the general exchange protocol: the microcontroller of each load generates a word in binary code which receives the general microcontroller $M C$, generates the word of the answer and sends to the microcontroller of the specific generator. Where $C_{0 i}=\frac{C_{B} \cdot B_{0 i}}{W_{0 i}}$.

corresponding generator.

Results and discussion. The developed mathematical model allows to receive a ratio for calculation of cost of base part of the electric power of the generator at dynamic change of level of the generated electric power. Also, model allows to study the influence of rather fast change of level of power of the generator and power of consumption of the electric power on change of cost indicators of economic system. This model will further allow the development of flexible dynamic tariffing for different types of Microgrid system. The dynamic influence of the parameters of the generating system and the levels of energy consumption on the economic indicators of the system is a significant factor in the rejection of the generally accepted hourly tariffs in the power grids of Ukraine due to its unprofitability. It is important to use the model of flexible dynamic tariffing, which will ensure adequate tariff price of electricity for consumers and producers.

\section{Conclusions.}

1. The analysis of scientific research shows that the regulation of prices in the energy market now occurs in most cases in statics, which does not allow for an adequate assessment of the dynamic change in the tariff price of electricity local systems Microgrid.

2. The urgent task is to create a dynamic model that combines, on the one hand, the energy performance of the energy generating system, and on the other - the economic performance of a closed macroeconomic system. Based on the equations of increase in the cost of electricity of the local Microgrid system, an electro - cost model is proposed, which allows to calculate the cost of the base part of energy depending on the level of electricity consumption.

3 . The proposed model allows the use of dynamic tariffing when changing the modes of operation of the generator, to provide an adequate price for consumers and producers of electricity. Also the algorithm of technical realization of calculation of cost of base part of energy at change of its volume in a dynamic mode is offered, on the basis of which the estimation of cost in a dynamic mode for a microgrid of various functional purpose can be carried out.

\section{ЛИТЕРАТУРА}

1. A.K. David, Y.C. Lee. Dynamic tariffs : theory of utilityconsumer interaction // IEEE Transactions on Power Systems, Vol. 4, No. 3, August 1989

2. Goran Koreneff, Anssi Seppala, Matti Lehtonen, Veikk:o Kekkonen, Er\& Laitinen; Jukka H\&li and Erkki Antila. Electricity spot price forecasting as a part of energy management in de-regulated power market // Energy Management and Power Delivery, 1998

3. Jie Chen, James S. Thorp, Robert J. Thomas, Timothy D. Mount. Locational Pricing and Scheduling for an Integrated EnergyReserve Market // System Sciences, 2003

4. M. Siahi, S. Porkar, A. Abbaspour-Tehrani-Fard, P. Poure and S. Saadate. Competitive distribution system planning model integration of $\mathrm{dg}$, interruptible load and voltage regulator devices // Iranian Journal of Science and Technology Transaction B: Engineering 34(6):619-635 December 2010

5. Steven Wong and J. David Fuller. Pricing Energy and Reserves Using Stochastic Optimization in an

Alternative Electricity Market // IEEE Transactions on Power

\section{Systems (Volume:22, Issue: 2 ) - 2007}

6. Joshua A. Taylor, Ashutosh Nayyar, Duncan S. Callaway, and Kameshwar Poolla. Consolidated Dynamic Pricing of Power System Regulation // IEEE Transactions on Power Systems (Volume:28 , Issue: 4 ) - 2013

7. Inam Ullah Nutkani, Wang Peng, Poh Chiang Loh, Frede Blaabjerg. Autonomous Economic Operation of Grid Connected DC Microgrid // 2014 IEEE 5th International Symposium on Power Electronics for Distributed Generation Systems

8. В.Я.Жуйков, д-р. техн. наук, проф., I.Ю.Бойко, асп. Застосування лінеаризованої електро-вартісної моделі для дослідження характеру перехідних процесів у ізольованій генеруючій системі // Енергетика: економіка, технології, екологія. 2018. № 4-с.41-49.

9. M. D. Bordo, «"Equation of exchange"». The New Palgrave: A Dictionary of Economics. № v. 2, pp. 175-77., 1987.

10. Дизельная электростанция 100 кВт расход топлива. URL: https://1kwt.com/

\section{REFERENCES}

8. V. Zhuikov, Dr. tech. sciences, prof., I. Boiko, Ph.D. student, Using of linearized electro - cost model for a transient processes research in isolated generating system // Energy: economics,

technology, ecology. 2018. № 4 - p. 4 - 49.

10. Diesel power plant $100 \mathrm{~kW}$ fuel consumption. URL: https://1kwt.com/ 